\title{
MIP-Guided Vascular Image Visualization with Multi-Dimensional Transfer Function
}

\author{
Ming-Yuen Chan ${ }^{1}$, Yingcai $\mathrm{Wu}^{1}$, Huamin $\mathrm{Qu}^{1}$ \\ Albert C. S. Chung ${ }^{1,2}$, and Wilbur C. K. Wong ${ }^{1,2}$ \\ 1 Department of Computer Science, and \\ 2 Lo Kwee-Seong Medical Image Analysis Laboratory, \\ The Hong Kong University of Science and Technology, \\ Clear Water Bay, Hong Kong \\ \{pazuchan, wuyc, huamin, achung, cswilbur\}@cs.ust.hk
}

\begin{abstract}
Direct volume rendering (DVR) is an effective way to visualize 3D vascular images for diagnosis of different vascular pathologies and planning of surgical treatments. Angiograms are typically noisy, fuzzy, and contain thin vessel structures. Therefore, some kinds of enhancements are usually needed before direct volume rendering can start. However, without visualizing the $3 \mathrm{D}$ structures in angiograms, users may find it difficult to select appropriate parameters and assess the effectiveness of the enhancement results. In addition, traditional enhancement techniques cannot easily separate the vessel voxels from other contextual structures with the same or very similar intensity. In this paper, we propose a framework to integrate enhancement and direct volume rendering into one visualization pipeline using multi-dimensional transfer function tailored for visualizing the curvilinear and line structures in angiograms. Furthermore, we present a feature preserving interpolation method to render very thin vessels which are usually missed using traditional approaches. To ease the difficulty in vessel selection, a MIP-guided method is suggested to assist the process.
\end{abstract}

\section{Introduction}

Prevention and treatment of vascular diseases can be improved if prompt and precise diagnosis can be performed with the aid of sophisticated vascular image visualization techniques. In practice, clinicians visualize the vascular images slice by slice or using the MIP. However, it is time consuming and difficult to realize the 3D structures. Less obvious structures may not be revealed because of other bright objects in the MIP. It cannot give the perception of depth and thus physical reality is lost as a result. Several recently published clinician studies [3] comparing DVR with surface rendering and MIP confirm that it is a more effective technique for angiography. Unlike other images, angiograms have several characteristics which make them difficult to visualize using traditional methods. First, the vessel intensity value is not fixed and is different in different types of images. The contextual objects may appear as bright structures and the intensity of vessels is suppressed after rescaling. Even the same vessel may have different intensity values in different parts. This makes it difficult to classify the vessels by mere intensity for visualization. Another challenge is the small vessels which are dim and obscure. They can be barely recognized even with the help of MIP. As the contrast of the vessels is small, it is difficult to separate simply by thresholding. Thinning or 
over-segmentation of vessels are common and noise is introduced into the final result. Furthermore, the vessels are usually accompanied by background structures which are not relevant but present an obstacle to classification. Removal of these contextual structures is difficult due to the potential overlapping between the intensity value intervals of different objects and vessels.

In this paper, we integrate filtering techniques into the visualization process under the framework of multi-dimensional transfer function. Segmentation and rendering processes are combined so that various visualization goals can be achieved by users with a higher flexibility. Besides, as we found that rendering of small vessels is problematic using conventional approaches, we proposed a new interpolation method to preserve the thin features. A MIP-guided selection method is suggested for vessel selection.

This paper is organized as follows: We introduce the previous work related to vascular image visualization in Section 2 and describe our framework which integrates both enhancement and visualization processes using multi-dimensional transfer function in Section 3. MIP-guided vessel selection method is explained in Section 4. A new feature preserving rendering approach is covered in Section 5. We demonstrate some experimental results in Section 6 and conclude our work in Section 7.

\section{Previous Works}

\subsection{Extraction and Enhancement}

Segmentation of vessels in medical images is important for diagnosis of the pathology of vessels. There are many promising segmentation methods developed, although none of them can outperform the others in every medical image modality. Recently, Kirbas and Quek [9] have done a survey on vessel extraction techniques. However, most of them are not fully automatic and require a certain degree of human interaction. For better visualization and diagnosis, vessel enhancement is another important issue. Different techniques for enhancement are reviewed in [18].

\subsection{Visualization}

Traditional approaches only show the planar cross-sections through the data volume. However, it is inefficient as only a small portion of the vessels is revealed in each slice. Curved Planar Reformation [7] tried to generate a cross-section through the centerline of the vessels. The correctness of the plane depends on the accuracy of the estimation of centerlines. Techniques based on the fusion of different rendering methods have been proposed. They use adaptive methods on the vascular image according to certain predefined criteria. In the two level volume rendering approach of Hauser et al. [4], different rendering techniques are selectively used for different parts of a 3D image. All the results of subsequent object renderings are then combined. Zhou et al. [19] developed a system to realistically render the region of focus, while data outside the region are rendered by NPR approaches. VesselGlyph [15], on the other hand, fused DVR and CPR in their solution according to the distance to the centerlines. 


\subsection{Multi-dimensional Transfer Function}

Multi-dimensional transfer function (MDTF) was first proposed by Levoy [11] who added the gradient as the second dimension to the transfer function in order to classify the boundaries of different classes of objects. Various approaches focusing on the use of the first and second derivatives in the design of the transfer function, such as semiautomatic transfer function generation [8] and manipulation widget design [10] have been investigated. Sato et al. [13] used more complicated classification rules to identify different structures and incorporated them into the feature space design of transfer function. Huang et al. [6] recently proposed a shaped-based approach for the segmentation of thin structures. Other related works like [5] tried to classify features using different types of parameter.

\subsection{Thin Structure Rendering}

The width of the vessels from different image modalities and resolutions can be quite different. Some of the small vessels can be as small as one voxel wide. In this case, displayed image is not satisfactory using the typical rendering approach. Aliasing effect and poor re-sampling results of the small vessels have to be handled. Dong et al. [1] proposed to find the presence of fine structures in a preprocessing stage by gradient estimation and render them with normal reconstruction. Their focus is on depiction of fine details and texture on a surface. The work of Sen et al. [14], although not directly related to fine structure rendering, tackled the aliasing problem of texture magnification using some sophisticated interpolation method. It is similar to the aliasing problem of small vessels.

\section{Multi-dimensional Transfer Function Design}

Owing to the complexity of angiograms, they cannot be effectively visualized using 1D transfer function. It is hard to determine the nature of the voxel by considering only the intensity. Vessels and other contextual structures may be misinterpreted in this mapping. Several ambiguous cases are summarized as follows.

First, two voxels with the same intensity are considered as the same class of objects in 1D mapping. However, it is possible that voxels with the same intensity represent different objects at different locations because of the overlapping of intensity interval of different object classes. Second, even if the voxels with the same intensity are proximate to each other, they may be of different classes. Due to the partial volume effect, a voxel may consist of different classes of structures(vessel and context). The voxels with the same intensity should not always be mapped to the same class. Lastly, in most cases, the intensity range of vessels is very small and overlaps with other classes. It is very difficult to distinguish the vessels in the intensity profile.

Therefore, we choose to extend the transfer function to higher dimension in order to resolve the uncertainty and limitations in the 1D approach. Previous work of Kniss et al. [10] indicated the importance of multi-dimensional transfer function in extracting materials and boundaries. Simply using derivatives as the second dimension of feature space [8] is not effective in our case. The gradient map of the angiogram can only show the boundary of different structures. The vessel response is relatively weak and 
cannot be distinguished from other contextual structures. This indicates that we should not only consider the boundaries of objects but also the contextual information or other higher level details of the objects in order to reveal the object of interest. In this paper, we focus on the problems arising from angiograms of different modalities and design a proper feature space for effective visualization of the vascular structures.

\subsection{Filtering Techniques}

As mentioned in Section 2.1, various kinds of vessel filtering techniques have been proposed mainly based on the characteristics of vascular structures. In this paper, we use a curvilinear structure filter and a line filter to assist the process of visualization.

Filter for Curvilinear Structure Vessels are considered as curvilinear structures by the filter and strong responses are generated at locations where similar structures are likely to be present. Among those filters in this category, vesselness measurement based on Hessian is adopted as it is widely used [12][13] and we found that it can reveal the tabular structures of vessels more precisely. The eigenvalues of the Hessian matrix are used to determine locally the likelihood of the presence of vessels. This helps discriminate the vessels from other contextual structures and recover those corrupted vessels. The Hessian matrix is given by

$$
H=\left(\begin{array}{ccc}
I_{x x} & I_{x y} & I_{x z} \\
I_{y x} & I_{y y} & I_{y z} \\
I_{z x} & I_{z y} & I_{z z}
\end{array}\right)
$$

where partial second derivative of image $\mathrm{I}(x)$ is represented by $I_{x x}, I_{x y}$, etc. For each point of the image, the second order structure of intensity variation is captured by the matrix and the corresponding eigenvalues and vectors which are represented by $\lambda_{1}, \lambda_{2}, \lambda_{3}$ and $e_{1}, e_{2}, e_{3}$ (where $\lambda_{1} \geq \lambda_{2} \geq \lambda_{3}$ ), can be computed. By analyzing the eigenvalues, different local structures can be predicted. The thin line structure of vessels results in a small $\lambda_{1}$ and large negative $\lambda_{2}$ and $\lambda_{3}$. To signify the line-like structure of vessels in the filter response, we use the line structure similarity measure suggested by Sato et al. [12] which is given by $L=f\left(\lambda_{1}, \lambda_{c}\right) \times \lambda_{c}$, where $\lambda_{c}=\min \left(-\lambda_{2}, \lambda_{3}\right)$ and

$$
f\left(\lambda_{1}, \lambda_{c}\right)= \begin{cases}\exp \left(-\frac{\lambda_{1}^{2}}{2\left(\alpha_{1} \lambda_{c}\right)^{2}}\right) & \text { if } \lambda_{1} \leq 0, \lambda_{c} \neq 0 \\ \exp \left(-\frac{\lambda_{1}^{2}}{2\left(\alpha_{2} \lambda_{c}\right)^{2}}\right) & \text { if } \lambda_{1}>0, \lambda_{c} \neq 0 \\ 0 & \text { if } \lambda_{c}=0 .\end{cases}
$$

By applying the filter, unrelated contextual structures can be removed. However, the performance depends on whether the filter scale is proper or not. It turns out that only vessels of a similar size can give a significantly high response. As we are dealing with the problem of small vessels, the smallest filter size is chosen. Users can selectively choose different filter sizes in their preferences for different visualization goals.

Filter for Line Structure Although the result of structural filter is pretty good, there are artifacts due to imperfect values of filter parameters or the variation of background tissue of the original image. These factors reduce the detectability of the small vessels. Uneven or unclear response and fragmentation can be found along the vessel. Refinements should be done on the response to connect those disconnected vessels and suppress those single bright voxels of noise. There are many line filters proposed which are 
quite similar in nature. The enhancement method proposed by Sun and Parker [16] is a simpler one and therefore can ease the burden of computation for real-time visualization. The idea is to find out the local mean of every line segment passing through the voxel of interest, while all the line segments are within a cubic kernel centered at the voxel. The local maximum mean (LMM) is defined as the maximum of the local means. By assigning the LMM to the previous response, the overall response is strengthened, especially for the vessel voxels.

$$
\operatorname{LMM}(\operatorname{vec} x)=\max _{j=1, \ldots, 13}\left\{L_{d}(\operatorname{vec} x, j)\right\}
$$

where

$$
L_{d}(\operatorname{vec} x, j)=\frac{1}{k} \sum_{l=-k / 2}^{k / 2} S(\operatorname{vec} x, j, l)
$$

However, the simplicity of the method comes with drawbacks. Bright vessels are widened and small vessels at bifurcation are blurred. Besides, the improvement is less significant for the dim vessels, therefore, an amendment is made to the original method.

To avoid the thickening of bright vessels, voxel intensity should not be raised significantly by a nearby single bright voxel. This can be done by lowering the intensity of other context voxels during the mean calculation. The context voxel can be identified by a threshold which is sufficiently lower than the intensity of the vessel. This can balance and suppress the increase of the final result due to the bright voxel. The original vessel is not affected as the highest mean does not change if it is connected with other bright voxels along the vessel. To suppress the context intensity, we can use a simple formula

$$
I^{\prime}(x)= \begin{cases}I(x) \times\left(\frac{T-I(x)}{T}\right)^{c} & \text { if } I(x)<T \\ I(x) & \text { if } I(x) \geq T .\end{cases}
$$

where $T$ is the threshold value and $c$ is the constant for the power function used in the intensity transformation. After applying the filtering methods described in the last two sections, we can get a clear filter response image which highlights and reveals the structure of the vessels in the original image. It can be added to the feature space of the vessel and participate in the transfer function and rendering process.

\subsection{Interface}

Recall that it is difficult to use a 1D transfer function to classify the vessels from the context due to the overlapping of intensity interval. As the intensity interval of vessels is small and not obvious compared with other background structures, it brings about difficulties in finding an optimal transfer function for visualization. Therefore, we use the multi-dimensional transfer function approach to ease the difficulties. Instead of using gradients [17] or derivatives [10], the aforementioned filtering response is treated as the second feature. To allow easy manipulation, we provide a $2 \mathrm{D}$ transfer function interface in which a user can define a transfer function by creating a polygonal region on the plane (Fig. 1(a)).

We can simplify the searching process by starting with a set of parameters. First, we define an approximate intensity range $\mathrm{R}$ of the vessels. The semi-automatic transfer function generation approach [8] can be a good initial guess which usually covers a larger interval than we need. Another range R', which is a smaller interval within R 


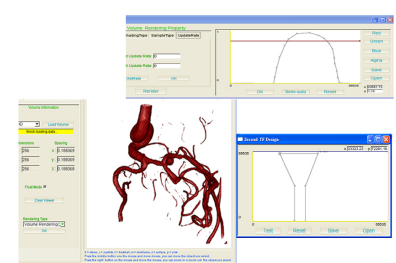

(a)

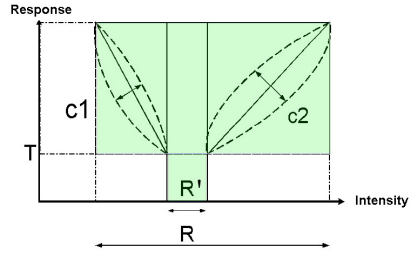

(b)

Fig. 1. (a) Interfaces of our system; (b) 2D transfer function Interface.

and can be more confidently classified as vessels, is defined. As a vessel would give a reasonably high response value, we can classify those voxels in the range $\mathrm{R}$ but not in range R' with a threshold defined by a curve or line. The transfer function is shown in Fig. 1(b). The curve is controlled by a threshold value $T$ and a constant $c$. Users can manually change these parameters or directly manipulate the shape of the function in order to get a better visualization result.

\subsection{Framework}

In a typical medical imaging system, the dataset is first preprocessed with different image processing tools and is subsequently put into the pipeline of visualization. This separated architecture reduces the users' interactivity in the course of finding a proper view for their visualization goals. Besides, the result of image processing cannot be accurate or proper for visualization without the interactive adjustments from users. For example, the preprocessed image may be suitable for visualizing large structures but not tiny vessels in certain regions. This indicates the need for better integration of both processes in order to accomplish the visualization goals of different users. Fang et al. [2] suggested a transfer function model in this spirit to fuse the processes. We adapt their hybrid approach for our purpose (Fig. 2). Instead of applying the filtering operations directly to the displayed image, the operations are performed on the responses. We consider the responses as the output of the image-based transfer function which is defined by F:I $\rightarrow$ I' where $F=f_{n} \otimes f_{n-1} \otimes \cdots \otimes f_{1}$

As users can interactively change the parameters of the filter, it provides higher flexibility. Without this, the result would be affected by poor preprocessed results. To avoid deterioration of performance due to filtering processes, the original method tries to relieve the computational cost by restricting the processes only on visible regions and voxels used for rendering. In our case, we restrict them by the first dimension of the transfer function. As vessels occupy only a small intensity interval and region, which are much less than $10 \%$ of the total number of voxels, this allows the process to be done more efficiently in real-time.

\section{MIP-Guided Selection}

The visualization result depends on viewers' concerns and therefore a proper user selection is a critical starting point for conveying results in an expected way. However, it is difficult to specify a region of interest in a volume data using a 2D interface. Instead 


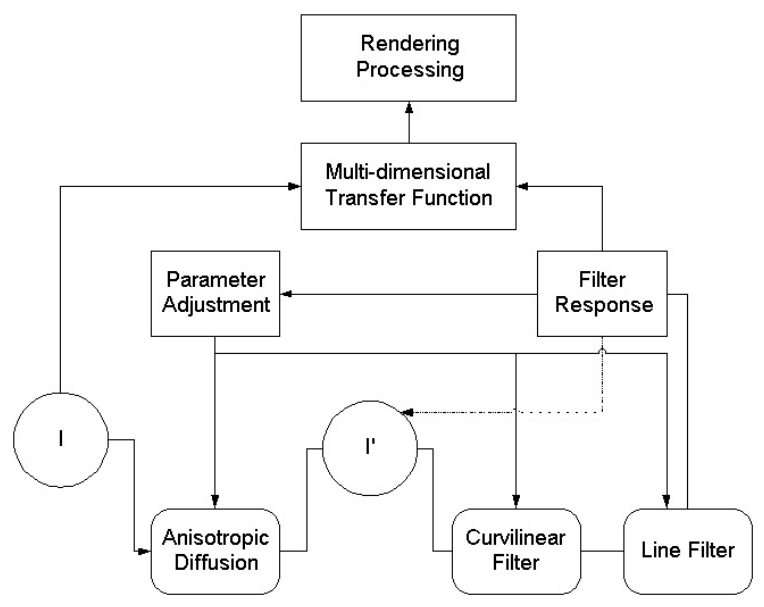

Fig. 2. A diagram of the visualization framework.

of performing the selection on each slice, we use the MIP to assist the process as it can provide useful information about the location of vessels. The idea is that the small vessels are usually projected on the MIP due to the relatively high intensity, but not on the direct volume rendered images. By choosing pixels belonging to vessels on the MIP, the corresponding voxels in the volume can be selected. Conventional region growing techniques can be applied to the selected voxels and a proper region which can capture the vessels is defined.

\subsection{Depth Encoding}

Vessels which are spatially close and have similar intensity values can lead to ambiguity. Without the depth information, it is difficult to identify the vessels individually and they are perceived as a clutter of connected vessels (Fig. 3(a)). To solve this problem, we encode the depth information using a spectrum of colors. First, we generate a depth map according to the distance of the voxels from the viewing plane. The depth map is encoded with colors and then composited with the mono-color MIP. This allows users to identify different vessels which overlap in the MIP. In our experiment, we use a color spectrum between red and blue to deliver depth cues (Fig. 3(b)). The twisted vessels in the MIP can be identified clearly. Without the aid of depth encoding, it is difficult to classify a vessel in the fuzzy context. By considering the color difference, users can select a vessel at different depth levels and understand the connectivity between vessels.

\subsection{Modification on MDTF}

In addition to the scalar intensity and vesselness measure, the distance to the selected voxels should be considered. We add this as another parameter to the MDTF. The opacity is adjusted accordingly and is increase near the selected regions. This opacity modulation can reflect the selection of users by highlighting the region of interest. The structure of the selected vessel (Fig. 4) which is otherwise occluded by other vessels can be shown clearly in the result. Actually, colors can also be adjusted based on the same principle. This method is also very useful for rendering those thin vessels of interest. 


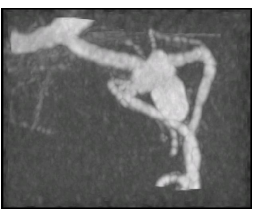

(a)

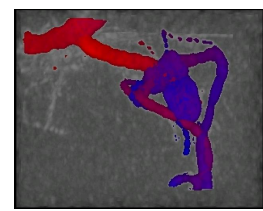

(b)

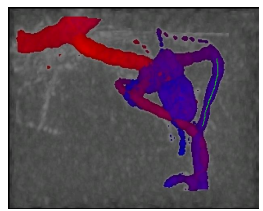

(c)

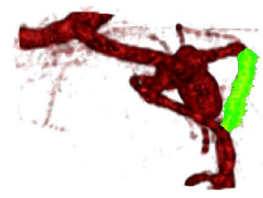

(d)

Fig. 3. MIP images: (a) Original; (b) Color encoded; (c) User selection; (d) Region growing.

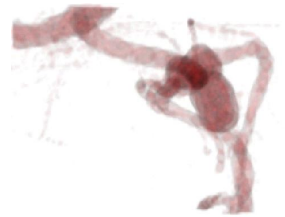

(a)

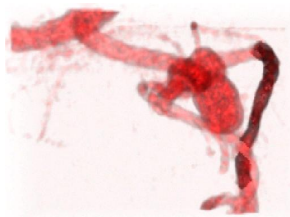

(b)

Fig. 4. Results of DVR: (a) Original; (b) Result of opacity modulation based on user selection.

\section{Feature Preserving Interpolation}

The intensity along the narrow and weakly connected part of the vessel is uneven and it is rendered in a ripple shape. Thin vessels may become invisible at certain viewing angles although they actually exist in the image. The reason for this artifact is that we cannot guarantee that a good sampling point can always be found along the rays from all angles. Although a ray passes through the vessel, the distance of the sampling point to the vessel varies from different viewing angles. We cannot guarantee that the sampling value is always close to the vessel's intensity. Therefore, part of a small vessel occasionally darkens or even becomes invisible from certain viewing angles. The artifacts can be attributed to the interpolation method used in the rendering process and can be reduced by increasing the sampling rate so that the chance of getting a representative sample point is increased. However, this is expensive and still cannot solve the problem of uneven density distribution. The basic problem is that the trilinear interpolation method in a typical rendering process has no idea of the existence of vessels (or more precisely, it does not know whether the voxels are connected within a cell). For example, the two diagonal voxels are weakly connected in the cell in Fig 5. The sampling value cannot truly reflect the situation if they are actually connected. This is a serious problem for small vessels. Inspired by Sen [14], we use a new interpolation method to preserve the connectivity of thin vessels.

\subsection{Interpolation method}

Our proposed rendering method takes weak connectivity into consideration. For the weakly connected vessels, we render them using a special interpolation method. Our objective is to ensure that the sampled intensity does not drop to a value lower than those of the connected voxels. A simple solution is to perform a linear interpolation between the two connected voxels for every connectivity. The intensity along the line 


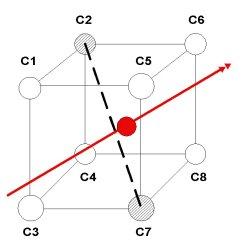

(a)

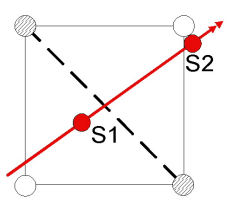

(b)

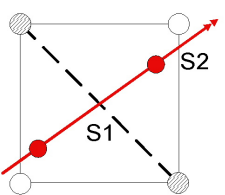

(c)

Fig. 5. Shaded voxels are the diagonal vessel voxels in a cell. The arrows are the rays passing through the cell and the circles on the rays are the sample points. A representative sample point $\mathrm{S} 1$ is found in (b) but not in (c). However, intensity of S1 in (b) still falls out of the intensity range of vessel after interpolation.

changes smoothly from one voxel to another. Then, the intensity of the sampling point is the value of the projected point of the sampling point on the line.

$$
I\left(P_{s}\right)= \begin{cases}\frac{I\left(c_{i}\right) \times \operatorname{dist}\left(P_{s}^{\prime}, c_{i}\right)+I\left(c_{j}\right) \times \operatorname{dist}\left(P_{s}^{\prime}, c_{j}\right)}{\operatorname{dist}\left(P_{s}^{\prime}, c_{i}\right)+\operatorname{dist}\left(P_{s}^{\prime}, c_{j}\right)} & \text { if } \operatorname{dist}\left(P_{s}^{\prime}, P_{s}\right) \leq R_{0} \\ I_{\text {Trilinear }}\left(c_{1}, \ldots, c_{8}\right) & \text { if } \operatorname{dist}\left(P_{s}^{\prime}, P_{s}\right)>R_{0}\end{cases}
$$

where $c_{1}, \ldots, c_{8}$ are the voxels of the cell, $P_{s}$ is the sampling point and $P_{s}^{\prime}$ is the projected point of $P_{s}$ on line $c_{i} c_{j}$ of the vessel. $R_{0}$ is the radius parameter used to control the width of the vessel. It should be a value large enough to ensure that at least one sampling point can be found in this cell when a ray passes through this vessel volume from any angles. For the volume outside the defined region, we can use the trilinear interpolation method.

\subsection{Connectivity}

We have to define the connectivity between voxels such that the vessels can be rendered accurately. If we only consider the intensity of the voxels, ambiguity may arise. For example, two voxels may be from two separate vessels or the same vessel. Therefore, we try to use the filter responses to predict the existence of connectivity. First, we identify the vessel voxels according to their response values. Then, we check the likelihood of connectivity by measuring the similarity of their structures. If these voxels have similar response value, we treat them as connected vessels. This criterion can be written like this:

Definition 1. Cell $C=\left[c_{1}, \ldots, c_{8}\right]$, where $c_{i}$ is a corner of $C$. For every pair of $c_{i}$ and $c_{j}$, find $R_{\text {diff }}(i, j)=\frac{\left|R\left(c_{i}\right)-R\left(c_{j}\right)\right|}{\min \left(R\left(c_{i}\right), R(c, j)\right)}$ where $c_{i}$ and $c_{j}$, are in diagonal. If $R_{\text {diff }}(i, j)<$ Threshold, connectivity exists between $i$ and $j$.

As the diagonal voxels may be connected indirectly with a voxel which is on the same edge with each connected voxel, the connectivity is preserved in the original interpolation method. Therefore, we can ignore these connectivity cases. There may be more than one connectivity in a cell. Performing interpolation for all the lines of connectivity is expensive and unnecessary. We only perform interpolation on the nearest edge to the sample point. For example, if the number of vessel voxels in a cell is quite a few (say, more than 4), we can assume that the vessels dominate the cell and the original interpolation can reveal the connectivity among them. In practice, we can get an improved result by considering only the case of 2-3 voxels. 


\section{Experimental Results}

In order to demonstrate that our approach can reveal the thin vessel structures and discard the unrelated structures and noises, two medical datasets are tested in our experiment. The first medical dataset is a 3D rotational X-ray angiographic image (3DRA) of size $256 \times 256 \times 256$. It shows the abdominal region of a patient. In the MIP (Fig. 6(a)), we find many thin vessels which are obscured by the other tissues. Fig. 6(b) and 6(c) show the results of applying $1 \mathrm{D} \mathrm{TF}$ and no suitable transfer function can be found after many trials. In Fig. 6(b) we adjust the transfer function to a higher intensity range so that noise is minimized. It can clearly show the large vessels but thin vessels are missed. In the right image, all the missing thin structures are revealed by broadening the intensity range. However, it is accompanied with noise and other tissues. This is due to the overlapping of the intensity intervals between vessels and soft tissues, which make it difficult to classify the vessels using 1D TF. We apply the MDTF with enhanced features to visualize the data (Fig. 6(d)). The feature response allows us to classify ambiguous voxels with similar intensity. By choosing a proper transfer function using the $2 \mathrm{D}$ interface, the result is better than the previous images. The noise, which has low response value, is greatly suppressed. Most of the small vessels are revealed clearly in the image.

In addition, a more complicated TOF MRA image is tested (Fig. 7). It is a $512 \times$ $512 \times 52$ brain image consisting of skull and brain matters. The vessels are small and dim and are embedded in different locations of the brain. Similar to the previous case, 1D TF cannot give a good result (Fig. 7(b) and 7(c)). Our result preserves more details of the vessels while keeping the noise at minimum. As the brain image is noisy and fuzzy (Fig. 7(a)), some regions of the brain with similar structures may be misinterpreted as vessels and introduce noise. It is impossible to remove them completely. However, the improvement is obvious in the comparison of our result with the original one.

Finally, we show the result of our feature preserving rendering method. Fig. 8(a) shows a small vessel extracted from the 3DRA data. The width of the vessel is about 1-4 voxels. In the conventional approach, vessels which are actually connected become broken or even invisible at certain viewing angles due to a poor sampling value being retrieved with the interpolation scheme (Fig. 8(b) and 8(d)). By using our method, the thin and weakly connected vessels can be seen clearly at any angle (Fig. 8(c) and 8(e)). As a representative sampling point is guaranteed to be found within a pre-defined radius, a strong connectivity is established without thickening the vessel.

\section{Conclusion}

This work presents a framework for visualization of vascular images. We integrate the enhancement process and visualization pipeline using multi-dimensional transfer function. Segmentation of small vessels is difficult and cannot be easily achieved by using 1D transfer function. The enhancement techniques help classify the curvilinear and line structures of vessels during the interactive visualization process. Thin vessels are highlighted and visualized. Also, we use a new feature preserving interpolation method to render the thin vessels. This ensures that the thin vessels can be seen clearly at any viewing angle and are not affected by poor sampling results of conventional interpolation methods. Moreover, we suggest a MIP-guided selection of vessel which helps users specify the structure of interest and deliver a better result based on the selection. 


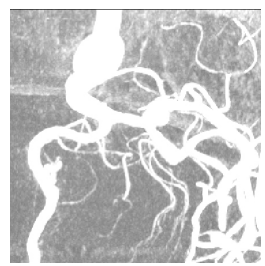

(a)

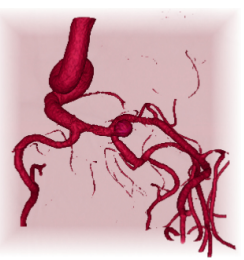

(b)

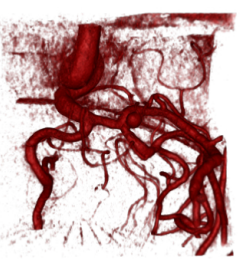

(c)

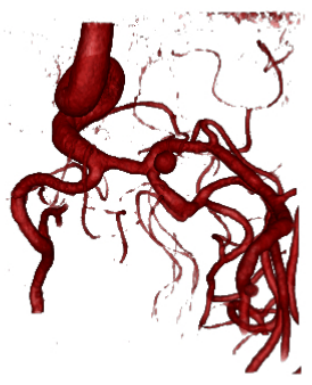

(d)

Fig. 6. 3DRA images: (a) MIP image; (b)(c) Results using 1D TF; (d) Result using MDTF.

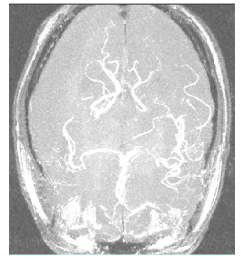

(a)

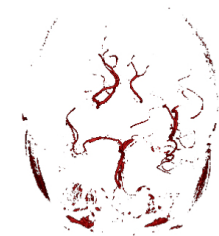

(b)

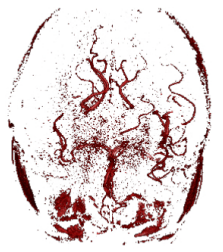

(c)

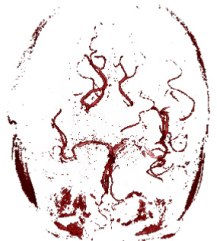

(d)

Fig. 7. Brain MRA: (a) MIP image; (b)(c) Results of using 1D TF; (d) Result using MDTF.

\section{Acknowledgments}

We would like to thank Dr. Simon C. H. Yu from Prince of Wales Hospital, Hong Kong for providing the medical data. This work is partially supported by RGC grant CERG 618705 and HKUST grant DAG 04/05 EG02.

\section{References}

1. F. Dong, G. J. Clapworthy, and M. Krokos. Volume rendering of fine details within medical data. In IEEE Visualization Conference Proceedings, pages 387-394, 2001.

2. S. Fang, T. Biddlecome, and M. Tuceryan. Image-based transfer function design for data exploration in volume visualization. In IEEE Visualization Conference Proceedings, pages 319-326, 1998.

3. E. K. Fishman. CT angiography and MDCT: Detection, characterization and staging of abdominal disease. RSNA, 2000. 


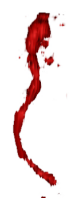

(a)

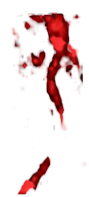

(b)

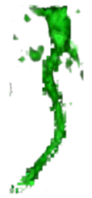

(c)

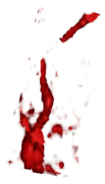

(d)

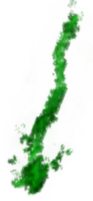

(e)

Fig. 8. Results using conventional approach (a) (b) (d) and feature preserving approach (c)(e).

4. H. Hauser, L. Mroz, Bischi, and E. Groller. Two-level rendering. pages 242-252, 2001.

5. J. Hladuvka, A. Konig, and E. Groller. Curvature-based tranfer functions for direct volume rendering. In Proceeding of Spring Conference Computer Graphics, pages 58-65, May 2000.

6. A. Huang, G. M. Nielson, A. Razdan, G. E. Farin, D. P. Baluch, and D. G. Capco. Thin structure segmentation and visualization in three-dimensional biomedical images: A shapebased approach. In IEEE Trans. on Visualization and Computer Graphics, volume 12, pages 93-102, 2006.

7. A. Kanitsar, D. Fleischmann, R. Wegenkittl, P. Felkel, and E. GrSller. CPR - curved planar reformation. In IEEE Visualization '02, 2002.

8. G. Kindlmann and J. W. Durkin. Semi-automatic generation of transfer functions for direct volume rendering. In IEEE Symposium On Volume Visualization, pages 79-86, 1998.

9. C. Kirbas and F. Quek. A review of vessel extraction techniques and algorithms. ACM Computing Surveys, 36(2):81-121, June 2004.

10. J. Kniss, G. Kindlmann, and C. Hansen. Multidimensional transfer functions for interactive volume rendering. IEEE Trans. on Visualization and Computer Graphics, 8(3), 2002.

11. M. Levory. Display of surfaces from volume data. IEEE Computer Graphics and Applications, 8(5):29-37, 1988.

12. Y. Sato, S. Nakajima, H. Atsumi, T. Koller, G. Gerig, S. Yoshida, and R. Kikinis1. 3D multi-scale line filter for segmentation and visualization of curvilinear structures in medical images. In IEEE Medical Image Analysis, pages 143-168, June 1998.

13. Y. Sato, C.-F. Westin, A. Bhalerao, S. Nakajima, N. Shiraga, S. Tamura, and R. Kikinis. Tissue classification based on $3 \mathrm{~d}$ local intensity structures for volume rendering. IEEE Trans. on Visualization and Computer Graphics, 6(2):160-180, 2000.

14. P. Sen. Silhouette maps for improved texture magnification. In Eurographics Graphics Hardware, 2004.

15. M. Straka, M. C. A. L. Cruz, A. Kochl, M. Sramek, E. Groller, and D. Fleischmann. The vesselglyph: Focus and context visualization in CT-Angiography. In IEEE Visualization Conference Proceedings, 2004.

16. Y. Sun and D. Parker. Small vessel enhancement in MRA images using local maximum mean processing. IEEE Trans. on Image Processing, 10(11), Nov. 2001.

17. F. Vega, P. Hastreiter, B. Tomandl, C. Nimsky, and G. Greiner. 3D visualization of intracranial aneurysms with multidimensional transfer functions. Bildverarbeitung fur die Medizin, pages 46-50, 2003.

18. W. C. S. Wong and A. C. S. Chung. A review on enhancement techniques in 2D and 3D vascular images. Technical Report(HKUST-CS06-02), The Hong Kong University of Science and Technology, 2002.

19. J. Zhou, M. Hinz, and K. D. Tonnies. Hybrid-focal region based rendering of medical data. Bildverarbeitung fur die Medizin, pages 113-116, 2002. 